\title{
A Phyletic Perspective on Cell Growth
}

\author{
Karl J. Niklas \\ Plant Biology Section, School of Integrative Plant Science, Cornell University, Ithaca New York 14853-5908 \\ Correspondence: kjn2@cornell.edu
}

Commonalities, as well as lineage-specific differences among bacteria, fungi, plants, and animals, are reviewed in the context of (1) the coordination of cell growth, (2) the flow of mass and energy affecting the physiological status of cells, (3) cytoskeletal dynamics during cell division, and (4) the coordination of cell size in multicellular organs and organisms. A comparative approach reveals that similar mechanisms are used to gauge and regulate cell size and proliferation, and shows that these mechanisms share similar modules to measure cell size, cycle status, competence, and number, as well as ploidy levels, nutrient availability, and other variables affecting cell growth. However, this approach also reveals that these modules often use nonhomologous subsystems when viewed at modular or genomic levels; that is, different lineages have evolved functionally analogous, but not genomically homologous, ways of either sensing or regulating cell size and growth, in much the same way that multicellularity has evolved in different lineages using analogous developmental modules.

The elementary parts of all tissues are formed of cells in an analogous, though very diversified manner, so that ... there is one universal principle of development... the formation of cells.

-Theodor Schwann

C ell size matters in four aspects of growth that are reviewed in this work: (1) cells must coordinate their growth in size with their physiological status; (2) at the cellular level, size affects the flow of mass and energy across membranes and, thus, physiological status by altering nutrient availability; (3) in addition, cell size influences the mechanical and dynamic properties of the cytoskeleton and how it operates during mitosis; and (4) the cells in multicellular organisms must fit together and work properly. Although these four topics are treated in separate sections, this review has two overarching themes.
The first is that cell "size" is not biologically meaningful unless it is measured and understood in the context of a cell's geometry, shape, ploidy, and location. Each of these aspects has received extensive scrutiny individually, but not collectively (see, however, Simova and Herben 2012). Size can be measured in a variety of different ways, for example, length, volume, surface area, dry mass, or DNA content. Each metric can be instructive. However, each can also alter our perception or redirect our attention regarding how a cell or an organism actually gauges its size. An additional concern is that a cell or organism may use different metrics to measure its size, depending on its external or internal circumstances. For example, when using cell volume or surface area as measure of cell size, it is important to recognize that geometry and shape are not the same thing. With the exception of the

Editors: Rebecca Heald, Iswar K. Hariharan, and David B. Wake

Additional Perspectives on Size Control in Biology: From Organelles to Organisms available at www.cshperspectives.org

Copyright (C) 2015 Cold Spring Harbor Laboratory Press; all rights reserved; doi: 10.1101/cshperspect.a019158

Cite this article as Cold Spring Harb Perspect Biol 2015;7:a019158 
K.J. Niklas

sphere, the shape and size of each geometric class of objects can be changed independently of the other. Thus, as a cell grows in size, it can (at least in theory) change its shape or geometry, or both simultaneously, and any or all such changes may provide a cell cues for registering its size. Ploidy must also play an important role in the measurement of cell size because statistically strong correlations exist among ploidy, nuclear volume, DNA content, cell size, minimum cell-doubling rates, and embryonic developmental rates (Cavalier-Smith 1982; Shuter et al. 1983; Gregory 2001). Ploidy can also influence gene expression profiles; for example, subsets of genes are reported to be either induced or repressed in ploidydependent ways within isogenic lines of yeast (Galitski et al. 1999). Finally, the location of a cell is an important variable of interest because it can provide a cell information about its status, for example, the concentration gradients of a morphogen. This aspect of cell growth is conceptually obvious when speculating about how uni- and multicellular organisms might register their size. The size of a cell in a multicellular organism might be based, in part or wholly, on the condition of the cells around it. In contrast, a unicellular organism might rely, at least in theory, on a mechanism registering only "size of self."

The second theme throughout this review is that a broad comparative phyletic approach is required to understand cell growth. Each of the following four sections shows that different lineages have evolved functionally analogous but not genomically homologous ways of either sensing or regulating cell size and growth, in much the same way that multicellularity has evolved in different lineages using analogous developmental modules (Niklas and Newman 2013). Certainly, some modules are conserved among otherwise different kinds of organisms. The land plants encode orthologs of the mammalian cell-cycle regulators retinoblastoma (RB) and p27/Kip1. However, in yeast, the functionalities of these regulators are taken over by Whi5 and Sic1. These and other examples suffice to show that evolution can take convergent pathways to cope with the biological demands shared by all organisms. For this reason, this work strives to identify generically func- tional modules in the mechanisms used to gauge cell size rather than to detail the genomically lineage-specific differences among bacteria, fungi, plants, and animals. The result precludes a detailed review of cell growth, but rather as broad an overview as page limitations permit.

\section{CELL SIZE AND PHYSIOLOGICAL STATUS}

As noted, cells must correlate nutrient availability with their growth in size (measured in terms of cell volume, surface area, or mass). The target of the rapamycin (TOR) signaling pathway provides one mechanism for connecting metabolic status and growth in size because its activation or suppression is coupled to cellular energy levels, nutrient availability, environmental stresses, and a range of growth factors, such as insulin and indole-3-acetic acid (Wullschleger et al. 2006; John et al. 2011). For example, the induced silencing of TOR in Arabidopsis results in the cessation of leaf growth, early yellowing caused by chlorophyll degradation, a reduction in the abundance of high molecular weight polysomes, and a decrease in the amount of soluble protein.

TORs are large proteins that share $40 \%-$ $60 \%$ sequence identity across such diverse eukaryotes as yeasts, worms, flies, mammals, algae, and land plants. Two TOR multiprotein complexes (TORC1 and TORC2) have been identified in all of the yeasts, mammals, and land plants thus far examined (Fig. 1A). TORC1 (which includes the LST8 protein or its homologs across all three lineages) regulates cell growth and metabolism by controlling protein translation, initiation, and transcription, ribosome biosynthesis, and mRNA stability. In turn, experimental evidence implicates TORC2 in the control of actin polarization and organization and, thus, growth-related cytoskeletal changes attending cell division or variations in geometry or shape during cellular ontogeny (Fig. 1A). High levels of nutrient availability activate the TOR pathway, whereas a variety of growth factors repress or activate TOR1.

Despite these and other similarities among diverse eukaryotes, significant differences exist in how TOR proteins operate in fungi, mammals, and land plants. For example, in mam- 
Phyletic Perspective on Cell Growth
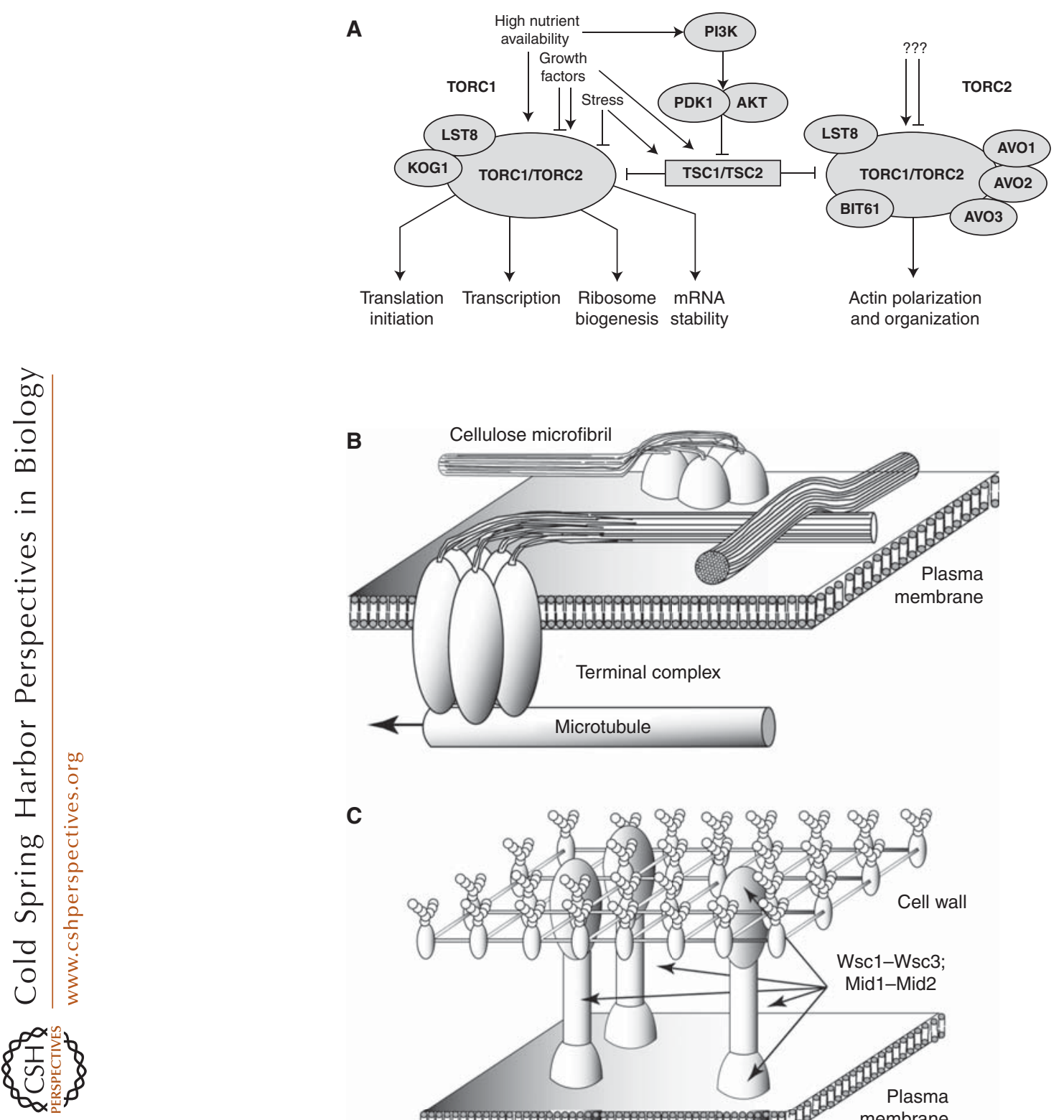

C

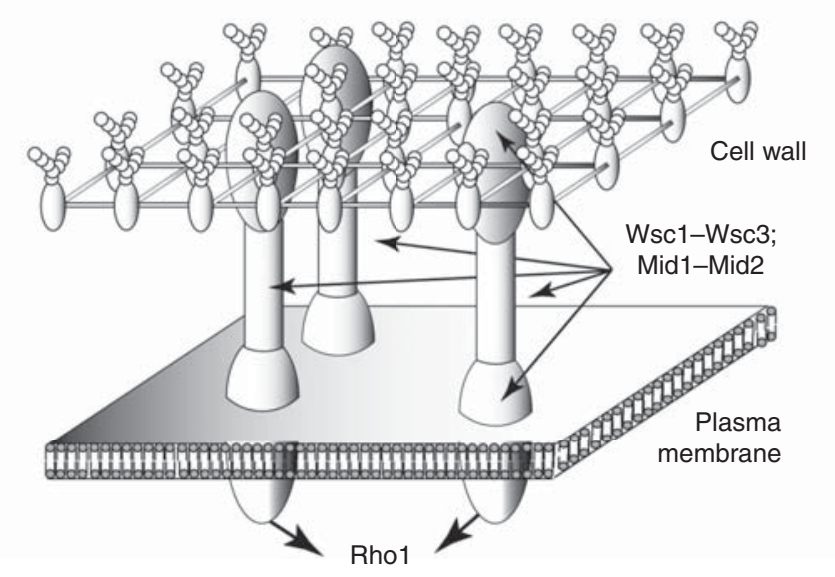

Figure 1. Schematics of the TORC1/TORC2, PI3K, and TSC1/TSC2 signaling pathways and portions of plant and yeast cell walls (CWs). (A) In conjunction with LST8 and other proteins, the TORC1/TORC2 pathway stimulates protein translation and transcription, ribosome biogenesis, messenger RNA (mRNA) stability, and actin polarization and organization. Both TORC1 and TORC2 are inhibited by the TSC1/TSC2 pathway that is, in turn, inhibited if growth factors trigger the PI3K pathway. (B) A portion of the plant plasma membrane (PM) showing two cellulose synthase terminal complexes (TCs) and three cellulose microfibrils (CMs). The TCs leave the CMs behind on the external surface of the PM as they are driven by microtubules. $(C)$ A portion of the yeast PM and CW associated with the yeast cell wall integrity (CWI) signaling pathway that transmits mechanical stresses (in the CW and PM) through a family of Wsc1 - Wsc3 and Mid1-Mid2 protein sensors to the Rho1 protein, which integrates signals from the PM membrane to govern $\beta$-glucan synthesis, actin organization, and polarized secretion. 
K.J. Niklas

mals, changes in insulin-like growth factors, or reductions in energy levels, or glucose or amino acid availability can activate the tuberous sclerosis complex (TSC), which is a negative regulator of TOR. Conversely, growth factors and increased nutrient availability can activate the phosphoinositide-3-kinase (PI3K) pathway. The importance of the PI3K pathway in terms of cell growth came from studies showing that overexpression of a dominant interfering allele of PI3K in the wing of Drosophila reduced cell size, whereas overexpression of the activated allele increased cell size (Leevers et al. 1996). Other studies have shown that PI3K inhibits TSC and, thus, stimulates the TOR pathway (reviewed by Kosma and Thomas 2002). In contrast, land plants appear to possess alternative TOR inhibitory pathways because they lack TSC homologs.

Among the land plants and in yeast (Baumberger et al. 2003; Levin 2005, 2011), the TOR complexes play an important role in the remodeling and reshaping of the cell wall, and the cell as a whole, because they influence protein synthesis and the polarization and orientation of actin (and, thus, aspects of cell division, such as the formation of the preprophase band and phragmoplast in land plants, both of which have actin components). For example, plant cell growth in size involves a turgor-driven cell wall expansion that requires a decrease in the mechanical yield stress of the cell wall, which is composed of a complex mixture of polysaccharides and proteins (Cosgrove 2005). One of the latter is the EXPANSIN protein family, which is believed to be involved in cell wall loosening (the addition of active expansin proteins to dead cell walls causes rapid wall extension, whereas overexpression of the EXP10 gene results in larger leaves with larger cells) in conjunction with auxin, which is one of the activators of TOR (reviewed by Bogre et al. 2013). The maintenance of cell wall integrity (CWI) and the degree of cell wall mechanical anisotropy depends on the synthesis and orientation of new cellulose microfibrils, which are constructed by cellulose synthase terminal complexes whose orientation is governed by the microtubule cytoskeleton (Fig. 1B). Therefore, the activation, suppression, or alteration of TOR can have important effects not only on the size of land plant cells, but also indirectly on their geometry and shape.

Yeasts have cell walls that are chemically and structurally very different from those of land plants. Nevertheless, TOR operates persona insignis comitans with the yeast CWI-signaling pathway (reviewed by Levin 2011). CWI transmits mechanical stresses (in the cell wall and plasma membrane) through a family of Wsc1Wsc3 and Mid1-Mid2 cell surface sensors coupled to a small G protein, Rho1, which integrates signals from the plasma membrane with a broad range of metabolic processes (Fig. 1C), including $\beta$-glucan synthesis, actin organization, and polarized secretion (Levin 2011). It is hypothesized that TOR inhibition, resulting from nutrient deprivation or physiological stress, results in plasma membrane stresses that are recognized by the Wsc and Mid sensors, which activate the CWI pathway. In turn, TOR repression of the CWI pathway likely prevents excessive cell wall deposition during vegetative growth. TOR2 control of the spatial component of cell division also likely affects various components of the CWI signaling pathway because the growth of yeast cells occurs at a discrete location, which requires a polarization of the underlying actin cytoskeleton. On a related note, the coupling of the Wsc1-Wsc3 and Mid1-Mid2 surface sensors to Rho1 in yeast is functionally analogous to the operation of animal integrins and plant integrin-like proteins, which directly or indirectly link to the cytoskeleton to transmit biomechanical signals that, in turn, transduce gene expression patterns affecting metabolism and cytoskeletal reorganization. This kind of module results in a positive/negative feedback loop that is ubiquitous in developmental systems.

\section{MASS AND ENERGY FLOW AND CELL CONTENTS}

The preceding draws attention to the relationship between signaling pathways and cell growth, nutrient availability, physiological stress, growth factors, and the biomechanics of the plasma membrane. However, the details known about the TOR, TSC, PI3K, CWI, and other signaling pathways are only part of a much larger story that 
involves the effects of cell size, geometry, and shape on the rates at which mass and energy move into and out of a cell. This aspect of cell growth is treated here in light of studies showing that cell growth, metabolism, and division rates decrease across (and often within) species as cell size increases (e.g., Kleiber 1947; Hemmingsen 1960; Banse 1976, 1982; Niklas 1994a,b; Tang 1995; Niklas and Enquist 2001).

Explanations for these inverse relationships differ among investigators, but considerations typically involve the size-dependent (scaling) relationships among cell surface area, volume, and dry mass (as measured by carbon or DNA content). The relationship between cell surface area and volume (and its effect on cell growth rates and division rates) has received the most attention because of the dictum that growth requires energy and, regardless of the form of energy garnered by a cell (radiant energy in plants; chemical energy in fungi and animals), the ability to harvest energy is some function of external surface area, whereas the metabolic requirement for energy is some function of volume.

This explanation is supported by the fact that, across vastly different lineages, cell growth rates decrease as the quotient of cell volume and surface area decreases. Why this is so can be gleaned by considering a set of objects differing in size, but maintaining the same geometry and shape, a condition that is illustrated by isolated protoplasts, lymphocytes, or even parenchyma plant cells. For these "Euclidean" objects, surface area is de minimis proportional to the square of some reference length, whereas volume is proportional to the cube of the same reference length. Because it scales as the $2 / 3$ power of volume, surface area will decrease as the $-1 / 3$ power of increasing volume (i.e., because $S \propto L^{2}$ and $V \propto L^{3}$, it follows that $S \propto V^{2 / 3}$ and $\left.\partial S / \partial V \propto V^{-1 / 3}\right)$. Accordingly, it pays to be small, but only if a cell exists in a 2/3 "Euclidean" space. If cell geometry and/or shape is free to change as a function of increasing volume, the $2 / 3$ and $-1 / 3$ scaling "rules" become irrelevant. For example, reliable measurements show that the rigid cell walls of many types of bacteria, unicellular algae, and fungi are geometrically similar to prolate or oblate spheroids, flattened disks, or cylinders. These measurements also show that, within each of these geometric classes, shape varies among species and occasionally within the same species, sometimes dramatically. Empirical studies show that these non-Euclidean cells have surface areas that scale nearly as the $3 / 4$ power of volume (Fig. $2 \mathrm{~A}$ ). The difference between $2 / 3$ and $3 / 4$ may appear trivial numerically, but it can be biologically significant for the exchange of mass or energy between a large cell and its environment.

Although the $3 / 4$ scaling relationship is more efficacious for absorption than the $2 / 3$ scaling relationship, it is still not good enough to countermand the potentially negative effects of increasing cell volume because surface area still decreases roughly as the $-1 / 4$ power of volume (i.e., $\left.\partial S / \partial V \propto V^{-1 / 4}\right)$. A perplexing mystery is why this is so. Theoretically, any cell can grow indefinitely in volume without a significant decrease in its surface area. For example, a cylindrical cell can increase indefinitely in length while maintaining a proportional curved surface area, provided that its radius remains constant (i.e., because $S=2 \pi r L$ and $V=\pi r^{2} L$, it follows that $S / V=2 / r=2 /$ constant). Some of the largest cells are cylindrical or have cylindrical components (e.g., the green siphonous alga Caulerpa and the nerve cells of the giant squid Architeuthis, both of which can be many meters in length). The question remains: why this is not so for all cells?

One explanation is that many cell types require a noncylindrical geometry and shape to maintain their appropriate function. But this does not explain why cell carbon content (dry mass) fails to increase one-to-one with increasing cell volume (Fig. 2B), or why, across uninucleated prokaryotic and eukaryotic cells, DNA content fails to keep pace one-to-one with increasing cell volume (Fig. 2C). This "cytoplasmic dilution" results in a decrease in the rate of the cell division as cell volume increases (Fig. 2D), albeit not invariably so (see Harris 1971). These correlations have been explained by postulating that DNA has two major functions unrelated to its protein-coding capacity: (1) the control of cell volume affected by the time required for DNA replication, and (2) the determination of nuclear volume by the overall bulk 

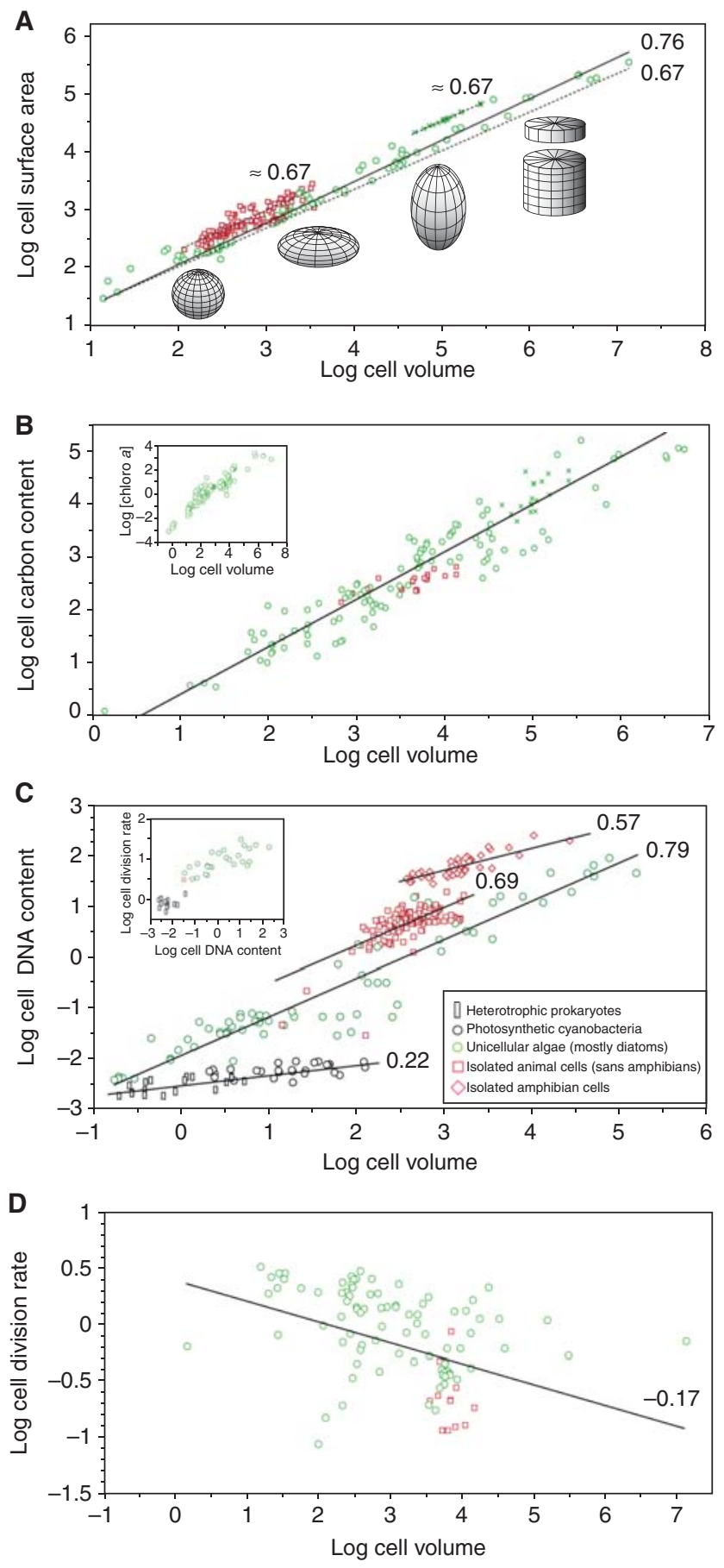

Figure 2. The log-log scaling relationships among cell surface area, volume, carbon content, and chlorophyll (chloro) a content, and cell-doubling rate. Regressions are reported based on model type II regression protocols. (A) The log-log relationship between surface area and volume. Dashed lines have slopes indistinguishable from those of Euclidean objects (slopes $=0.667 ; r^{2}=0.99 ; P<0.0001$ ); the solid line has a slope $\approx 3 / 4$ (slope $=$ $\left.0.76 ; r^{2}=0.98 ; P<0.0001\right)$. (B) The log-log relationship between cell carbon content (pg $\mathrm{C}$ per cell) and volume (and between chlorophyll $a$ content and cell volume; see inset). The solid line has a slope $<1$ (slope $=$ $\left.0.90 ; r^{2}=0.95 ; P<0.0001\right)$. (C) The log-log scaling relationship between cell DNA content and volume (and between cell DNA content and division rate; see inset). (Legend continues on following page.) 
of the DNA. In turn, cell growth rates are postulated to be determined by cell volume and the area of the nuclear envelope available for the nucleocytoplasmic transport of RNA that, in turn, depends on nuclear volume and, thus, on DNA content. During evolution, nuclear volume (and, thus, DNA content) would have to be adjusted for differences in cell volume to allow for biologically reasonable growth rates (Simova and Herben 2012). The great diversity of cell volumes and growth rates and, therefore, of DNA content among eukaryotes would result from a varying balance in different species between $r$-selection favoring small cells with rapid growth rates (and, therefore, low DNA C-values) and conditions permitting large cells with comparatively slower growth rates (and, therefore, permitting high DNA C-values) (Hessen et al. 2010). In multicellular organisms, cell size needs to vary in different tissues, which provides one possible explanation for differences in the ploidy of somatic cells resulting from polyteny and endopolyploidy; for example, the experimental manipulation of somatic ploidy, either increasing or decreasing ploidy levels, results, respectively, in an increase or decrease in the adult body size of Caenorhabditis elegans (Lozano et al. 2006). It is worth noting that all of these speculations and experimental observations resonate with the "energiden" hypothesis first proposed by Julius Sachs (1892), that is, each nucleus can metabolically control only so much cytoplasm (for a recent version, see Cavalier-Smith 1982).

\section{CELL SIZE, DIVISION, AND THE CYTOSKELETON}

A third aspect of cell growth among algae, land plants, animals, and fungi involves the timing and location of cell division. Across these diverse organisms, cell division is correlated with the dimerization and activation of cyclins and cyclin-dependent kinase (CDK) pairs. Cyclin/ CDK complexes phosphorylate target proteins to activate a sequence of events that advance the cell cycle through the mitosis phase. Although they are not well understood in plants, most of these regulators are highly conserved (Cross et al. 2011). For example, plant genomes possess orthologs of mammalian cell-cycle regulators, such as RB and p27/Kip1. In budding yeast, Whi5 and Sic1 function as RB and p27, although these proteins appear not to be phylogenetically related (Cross et al. 2011). Thus, the regulatory network and dynamics of the cell cycle are conserved evolutionarily, although the protein components operating within these networks are not. Consequently, generalized models for the eukaryotic cell cycle (featuring positive feedback loops driving the irreversible advancement of the cell cycle from one stage to the next) are conceptually and biologically acceptable, even if they identify taxonomically idiosyncratic components (Csikász-Nagy et al. 2006; Roeder 2012).

Importantly, the timing, as well as the location, of cell division can be the consequences of cell size and geometry as illustrated by current models for cell division in some bacteria, fission yeast, and land plants - many of which give the appearance of a Turing reaction-diffusion system. For example, the site of cell division in the rod-shaped bacterium Escherichia coli is forecast by at least three different mechanisms, each of which inhibits the polymerization or the function of the protein FtsZ, which is responsible for nucleating cell division throughout all of the bacteria thus far examined. The discovery

Figure 2. (Continued) C-DNA content increases with increasing cell volume, but not at a commensurate (oneto-one) rate for heterotrophic prokaryotes and cyanobacteria (slope $=0.22 ; r^{2}=0.67 ; P<0.0001$ ), unicellular algae (slope $=0.79 ; r^{2}=0.89 ; P=0.0001$ ), or cells isolated from amphibians (slope $=0.57 ; r^{2}=0.73 ; P<$ 0.0001 ) or other animals. Across prokaryotes and unicellular algae, cell division rates increase to a limit with increasing DNA content (see inset). (D) The log-log relationship between cell division rate and volume. The solid line has a slope significantly $<1$ (slope $=-0.17 ; r^{2}=0.16 ; P<0.0001$ ). (see inset for key to taxa.) Original units are in $\mu \mathrm{m}$ and pg. (Data taken from Williams 1964; Eppley and Sloan 1966; Mullin et al. 1966; Mandels et al. 1968; Taguchi 1976; Olmo 1983; Shuter et al. 1983; Langdon 1987, 1988; Agustí 1991; von Dassow et al. 2006, 2008; Connolly et al. 2008.) 
K.J. Niklas

of one of these mechanisms, which measures the size of E. coli cells as gauged by cell length (and, thus, cell shape), can be traced to the identification of the minicell mutation, which eventually led to the discovery of the three-gene operon, the $\min C D E$ locus (de Boer et al. 1989). This involves the FtsZ-inhibiting protein MinC, which is driven to oscillate between the ends of the cell by two proteins, MinD and MinE (Fig. 3A). Each cycle, which takes roughly 1-2 min, results in a time-averaged MinC concentration, which reaches its minimum at the cell's midpoint (Rothfield et al. 2005; Lutkenhaus 2008). As the cell grows in length, the concentration of MinC decreases below a critical value that inhibits FtsZ, at which point (and time) FtsZ polymerizes and activates cell division.

This MinCDE mechanism is operationally similar to the Pom1 mechanism, which operates in the fission yeast Schizosaccharomyces pombe (Fig. 3B). Pom1 is a kinase that inhibits yeast $\mathrm{G}_{2} / \mathrm{M}$ until cells reach a critical length. It is transported to the cell poles, where it accumulates and, subsequently, diffuses to produce a gradient that reaches its minimum at the cell's midpoint. In small (=short) cells that are in early $\mathrm{G}_{2}$, Pom1 inhibits Cdr1/Cdr2, which prevents entry into mitosis, by inhibiting Weel that, in turn, inhibits Cdc2(Cdk1) kinase activity, which drives mitosis and, thus, determines the length of $\mathrm{G}_{2}$ (Martin and Berthelot-Grosjean 2009; Moseley et al. 2009). As the cell grows and increases in length, the midlength concentration of Pom1 drops below a critical level, which releases the Cdr1/Cdr2 $\rightarrow$ Wee $1 \rightarrow$ Cdr1/Cdr2 cascade, resulting in cell division. Although cell length is registered to regulate mitosis, the Pom1 mechanism does not operate in isolation. Cells in which Cdc2 phosphorylation is prevented (and thereby bypassing Pom 1 regulation), nevertheless, still exert control over "normal" cell length, albeit with a much broader size frequency distribution. It is worth noting that the MinCDE and Pom1 mechanisms operate in an analogous manner to the diffusible heterocyst inhibitor system in the filamentous cyanobacterium Anabaena (Wilcox et al. 1973).
The mechanisms responsible for the location and orientation of the future cell wall in land plant cells and animals are much less well understood, although it is almost certain that these mechanisms gauge cell size and geometry by methods that include measuring mechanical forces. Elegant experiments using the effects of centrifugation on both haploid and diploid land plant cells have shown that the position of the interphase nucleus (which prefigures the preprophase band and the phragmoplast) establishes the location of the future division plane (Mineyuki and Gunning 1990; Murata and Wada 1991). Based on these and other experiments, a recent model for land plants proposes a microtubule (MT) length-dependent force-sensing system that permits the cytoskeleton to position the nucleus (and, thus, the preprophase band) into a biomechanically equilibrated position (Besson and Dumais 2011). If the nucleus in interphase is positioned artificially offcenter, the MTs radiating from it, outward to the cell cortex, are envisioned to recenter the nucleus based on differences in the tensile forces generated among the MTs differing in length (Fig. 3C,1-3). Shorter, as opposed to longer, MTs would be favored collectively to achieve the equilibrium configuration, which would automatically coincide with the minimal area plane that is classically thought to trigger the location of the preprophase band, which prefigures the location and orientation of the new cell wall. Cells that are too large would have MTs that would be unable to tether the nucleus to some of the cell wall's facets; cells that are too small would have MTs experiencing compressive rather than tensile forces (Fig. 3C,4,5). A similar length-dependent force-sensing system may operate during C. elegans embryogenesis because the centrosomes in embryonic cells treated with microtubule-depolymerizing drugs fail to reach the cell center and the division plane is repositioned (Strome and Wood 1983).

That biomechanically induced mechanical stresses may be involved in plant cell wall orientation is consistent with many observations, which are summarily rendered by "Errera's rule" (Errera 1888). The simplest plant cells are those that constitute parenchyma. These have 
A

1.

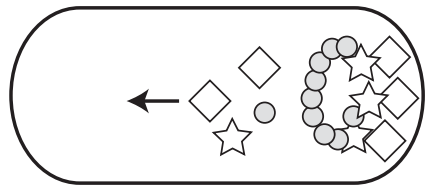

2.

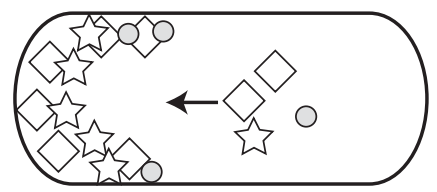

3.

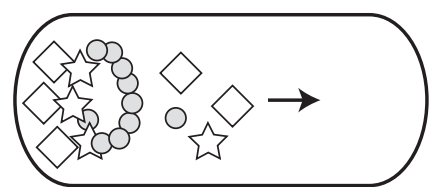

4.

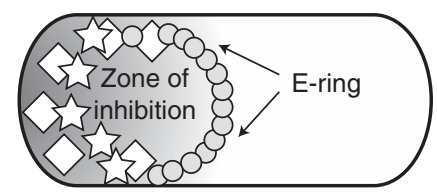

药 MinC

$\triangle \operatorname{MinD}$

MinE
C
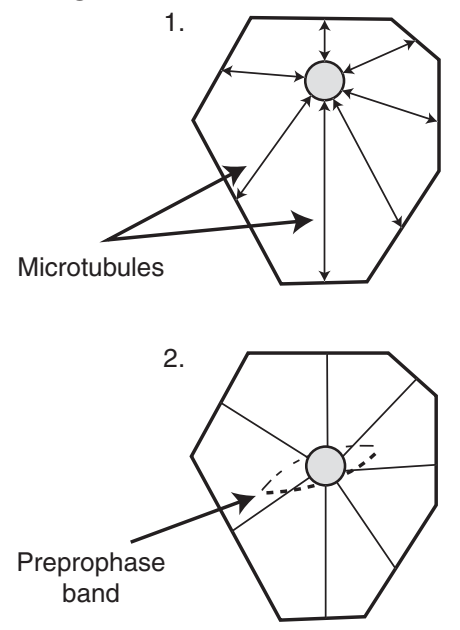

3.

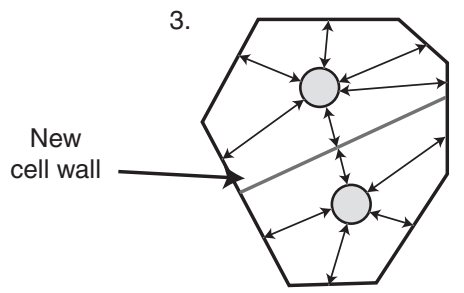

B

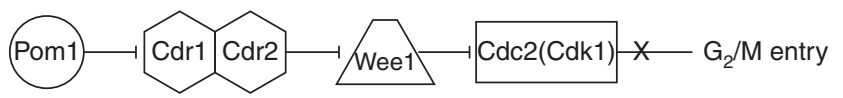

4

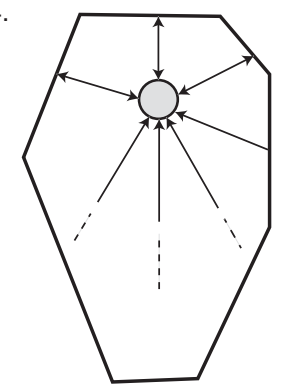

5.

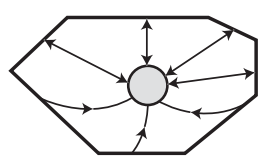

Figure 3. Schematics of models for the location of the future cell walls in E. coli, fission yeast, and land plant cells. (A) The MinCDE oscillation model for E. coli, in which the MinCDE polar zone begins assembling at one cell end, grows midcell (1), assembles at the opposing cell end (2) where it disassembles, releasing MinC, MinD, and MinE molecules, shrinks back to the cell end (3), and finally releases MinE from the E-ring (4) (adapted from Rothfield et al. 2005; Fig. 1). (B) The Pom1 gradient model for fission yeast, in which Pom1 from the cell ends diffuses toward the midcell, where it inhibits the $\mathrm{Cdr} 1 / \mathrm{Cdr} 2 \rightarrow$ Wee $1 \rightarrow \mathrm{Cdr} 1 / \mathrm{Cdr} 2$ cascade that, in turn, prevents $\mathrm{G}_{2} / \mathrm{M}$ entry (upper diagram). As the cell grows in length, the midcell Pom 1 concentration drops below a critical threshold on which the Cdr1/Cdr2 $\rightarrow$ Wee $1 \rightarrow \mathrm{Cdr} 1 / \mathrm{Cdr} 2$ cascade is operative (lower diagram). $(C)$ The microtubule (MT) force-sensing model, in which the tensile strains in the MTs tethering a nucleus in plant cells are adjusted (1), thereby positioning the nucleus at an equilibrium location where the preprophase band (2) prefigures the location and orientation of the future cell wall (3). Cells that are too large or too small have MT cytoskeletons that are unable to properly locate the nucleus (4 and 5). 
K.J. Niklas

thin primary walls that are hydrostatically inflated. The turgor pressure exerted against the walls of these cells is more or less uniform; that is, the stresses resulting from turgor pressure within cell walls are uniform, both within each cell, and among neighboring cells. However, at the vertices created by adjoining cells, opposing tensile stresses are resolved into additional stresses acting in the radial direction on the angle of each vertex according to its angle size. In theory, the tensile stresses in walls at $180^{\circ}$ should be equal and opposite and, thus, this angle experiences no additional radial stress from the resolution of the opposing tensile stresses in the two intersecting walls. However, these tensile stresses are resolved into progressively larger radial stresses as the angle of a vertex decreases, reaching their maxima as the angle approaches $0^{\circ}$. Because these additional radial stresses are correlated directly to the size of the angle, stresses reach mechanical equilibrium at equiangular vertices. The observation that the vertices in the region of isodiametrical expansion can act as cellular pivots for wall rotation between successive divisions (so as to coincide with cellular mechanical equilibria) provides some evidence for the biomechanical regulation of cell shape.

At least one component is missing from all of the currently available cell-size-monitoring models: the almost-universal relationship between ploidy and cell size (see Fig. 2D). Cells must be able to monitor their ploidy levels and integrate this information in whatever cell-sizemonitoring mechanisms they use. At least two monitoring-integration models appear reasonable: (1) a mechanism that synthesizes a specific component whose concentration is dependent on ploidy, or (2) a mechanism that measures the amount of a specific component against ploidy levels (Fantes et al. 1975). The size of a critical burst of some kind of transcription product would be a measure of a cell's ploidy and, thus, an example of the first of these two mechanisms. A protein binding to selected portions of the genome, maintained at a constant concentration, and measured by the number of bound sites would provide an example of the second mechanism.

\section{COORDINATING CELL AND ORGANISM SIZE, AND CONCLUDING REMARKS}

The fourth and last aspect of cell growth treated here is the measurement of size within a tissue, organ, or entire organism. As noted, cells have to achieve appropriate sizes, geometries, and shapes for their functional roles for an organism to operate properly. Yet, among all the topics treated in this review, this aspect of growth and development is perhaps the least well understood.

Evidence gathered over many years does show that plants and animals can regulate the overall size of their structural components (i.e., tissues, tissue systems, organs, and organ systems) by monitoring the absolute size of these components, in addition to gauging cell size and number within each (Satina et al. 1940; Fankhauser 1945a,b; Foard and Haber 1961; Vervoort et al. 1999; Tsukaya 2003; also see Day and Lawrence 2000; Mizukami 2001; Potter and Xu 2001). As a consequence, the functional components of multicellular organisms typically grow to their characteristic size and shape even when experimentally manipulated to change cell number or size, or when they are irradiated to abort cell division entirely (Fig. 4). Thus, contra Theodor Schwann's proclamation omnis cellula e cellula, the development of multicellular organisms seems to resonate more with Heinrich de Bary's dictum die pflanze bildet zellen, nicht die zellen bilden pflanzen. To be sure, alterations of genome size via polyploidization or endoreduplication can reset the checkpoint for the overall size of a cell, tissue, or organ presumably by changing epigenetically regulated differential gene expression in ways that, nevertheless, substantively differ between plants and animals (Mizukami 2001). However, it seems that the cellular components within multicellular organisms can "read" their size as they develop, either in reference to their local or global position.

In this context, it is important to recognize that a distinction must be made between "measuring size" in a unicellular versus a multicellular organism. In a cell's division cycle, across very diverse organisms, the process of measuring cell size appears to be triggered near the 
A

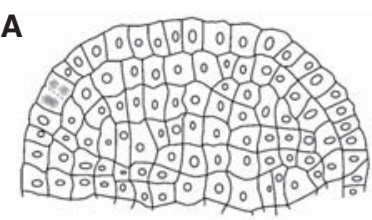

$2 \mathrm{n}, 2 \mathrm{n}, 2 \mathrm{n}$

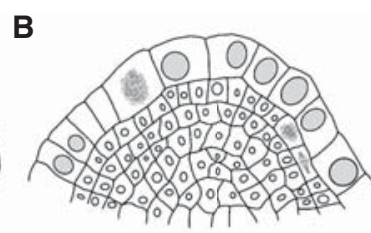

$8 \mathrm{n}, 2 \mathrm{n}, 2 \mathrm{n}$

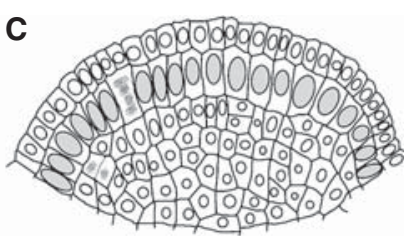

$2 n, 4 n, 2 n$
D

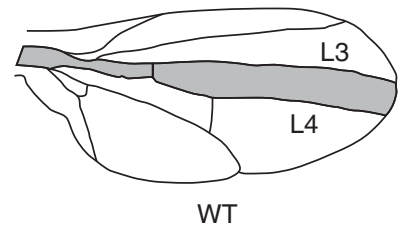

$\mathrm{E}$

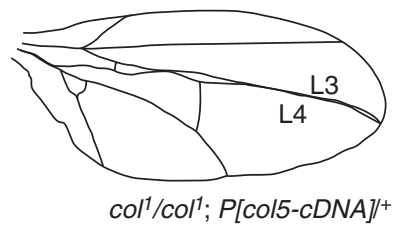

$\mathbf{F}$

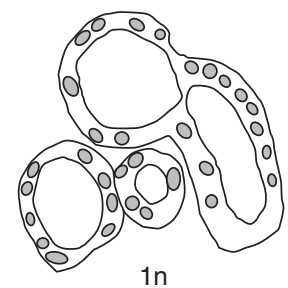

G

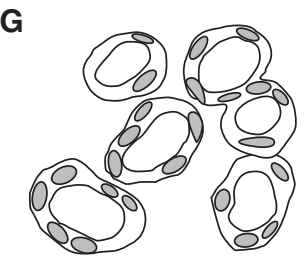

$2 n$
H

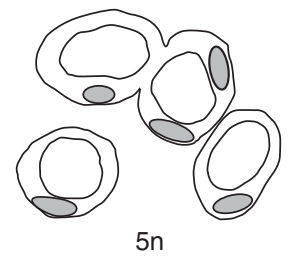

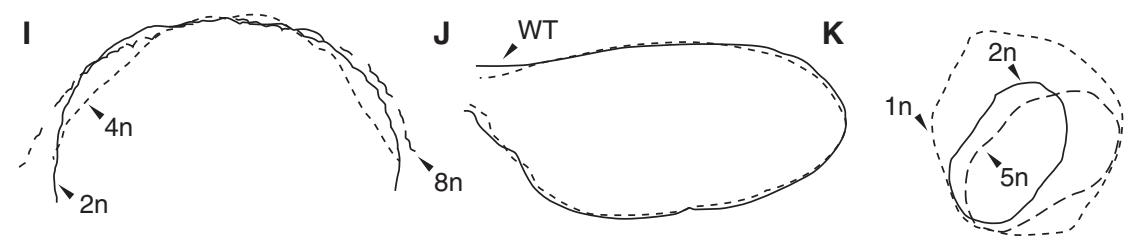

$\mathbf{L}$

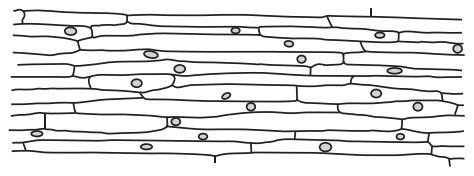

M

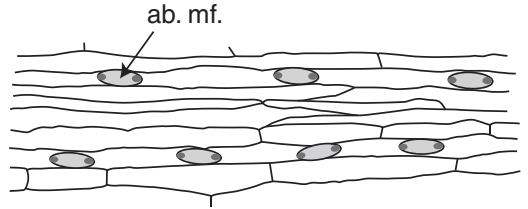

Figure 4. Comparisons of plant and animal organs differing in experimentally induced ploidy, or cell number or size, but conserving overall size. All organographically comparable diagrams are drawn to the same scale. $(A-C)$ Median longitudinal sections through periclinal chimeras (2n, 2n, 2n = normal) of Datura shoot apices drawn to the same size (shaded areas denote nuclei; redrawn from Satina et al. 1940; Table 1). (D,E) Drosophila wings of wild type (WT) and the $\mathrm{col}^{1} / \mathrm{col}^{1} ; P[c o l 5-c D N A] / /^{+}$double mutant lacking the sector area between longitudinal veins L3 and L4 (sector area denoted by shaded area; redrawn from Vervoort et al. 1999; Fig. 1A,B). (F-H) Transsections through pronephric tubules from $1 \mathrm{n}, 2 \mathrm{n}$, and $5 \mathrm{n}$ Triturus viridescens larvae (shaded areas denote nuclei; redrawn from Fankhauser 1945b; Fig. 1). (I-L) Superimpositions of the outlines of Datura shoot apices (see A$C$ ), Drosophila wings (see $D-E$ ), and pronephric tubule lumens (see $F-H$ ) differing in ploidy. $(L, M)$ Epidermal cells of the first foliage leaves of untreated $(L)$ and $\gamma$-radiated wheat seedlings ( $M$; ab. mf., aborted mitotic figure) (redrawn from Foard and Haber 1961; Figs. 9 and 10). The first leaves of both seedlings are comparable in size (not shown). 
K.J. Niklas

beginning of the $\mathrm{S}$ or $\mathrm{M}$ phase as a cell approaches its characteristic size (for a review, see Mitchison 2003). Among unicellular organisms, the mechanism responsible for controlling size maintains a reasonably uniform size frequency distribution from one generation of cells to another (at least as gauged by laboratory studies of cell cultures). However, many of the cells in plants and animals pass through a limited number of cell cycles before they differentiate and stop dividing. Consequently, preventing significant divergence in cell size from one generation to another is irrelevant, whereas achieving cell sizes appropriate to the locations and functions of cells becomes paramount.

With this caveat in mind, the developmental mechanisms that coordinate cell growth in size, division, and death within the various multicellular compartments of plants and animals appear to share similar, albeit not identical, developmental modules. One of these modules is a total size checkpoint mechanism. In the simplest case, this module could by analogous to that used by bacteria and fission yeast to measure cell size (see Fig. 3A,B). That is, a mechanism using concentration gradients established by substances diffusing along each of the principle axes of symmetry as, for example, the participation of concentration gradients of indole-3-acetic acid and other phytohormones in angiosperm leaf morphogenesis (see Benkova et al. 2003; Hay et al. 2006). However, a number of experiments indicate that disruption of pattern formation or cell proliferation can deregulate overall organ size. For example, ectopic expression of either the Hedgehog or Decapentaplegic protein in the Drosophila wing disk can produce duplicated wing structures (Capdevila and Guerrero 1994), mutation of the Drosophila lats gene results in greatly enlarged larvae (Xu et al. 1995), and etol and ctrl gene mutations result in smaller-than-normal organs because of reduced cell numbers and size in Arabidopsis (Ecker 1995). Mizukami and Fischer (2000) examined the function of ANT in the control aspects of shoot development of Arabidopsis and

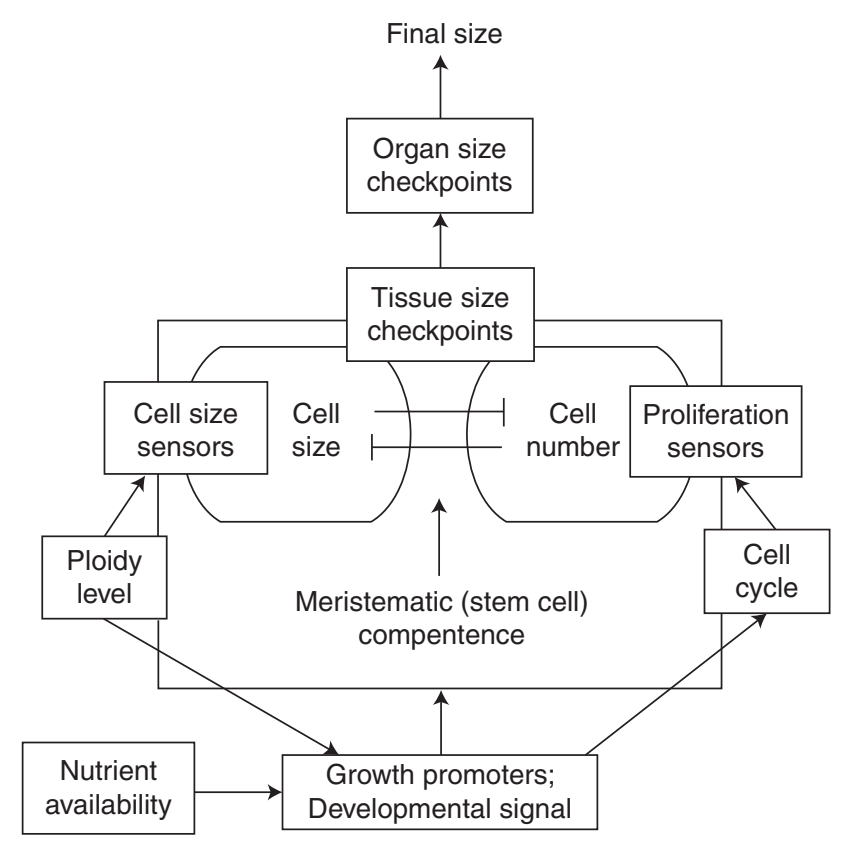

Figure 5. Schematic of a hypothetical network composed of generic modules regulating final organ size by sensing and regulating cell size and number (involving cell ploidy levels and cell-cycle status) in coordination with monitoring meristematic (stem cell) competence. Each module in the network is hypothesized to have an analog in any multicellular organism. Ancillary modules regulating cell differentiation and feedback loops are not diagramed. 
Nicotiana and report that ant mutations reduced the size of all floral parts and leaves as a result of decreased cell number, whereas 35S::ANT expression enlarged organs without altering their overall appearance by increasing cell number. Thus, the identification of a "total organ size checkpoint" is only part of a much larger and complex developmental system.

In view of our understanding of how organisms perceive, measure, and regulate the size of their multicellular components, it seems reasonable to suppose that multiple modules are integrated to coordinate overall growth in size. These include a module regulating the duration of meristematic (stem cell) competence, as well as modules monitoring cell size and number throughout tissue- and organogenesis (Fig. 5). Some of these modules likely use ploidy levels and cell-cycle status as sensors and/or regulators, whereas others likely involve growth regulators and a variety of developmental signals. Comparative studies of multicellular bacteria, fungi, animals, and plants indicate that some of these components may be highly conserved across a broad spectrum of organisms (e.g., the TOR signaling pathway), whereas others are likely to be specific to one or a few particular lineages (e.g., ANT functionality in angiosperms). For this reason, a broad comparative phyletic approach is required, one that identifies how each module generically functions within a larger system, as well as how each component is genomically specified. In this kind of global system, it is futile to label any module as the "master cell growth" module because this system contains recursive negative and positive feedback loops. This injunction is relevant to understanding even simple bacterial cells because modeling and experimentation reveal the equal importance of the coupled effects of the global physiological status of cells (as revealed by the activity of their gene expression profiles) and DNAbinding transcription factors and other cell regulators (Berthoumieux et al. 2013).

\section{ACKNOWLEDGMENTS}

The author thanks Drs. Rebecca Heald, David Wake, and Iswar Hariharan for inviting this re- view and Dr. Dominick J. Paolillo, Jr. and Randy Wayne (Cornell University) and two reviewers for their comments and suggestions. Funding from the College of Agriculture and Life Sciences (Cornell University) is gratefully acknowledged.

\section{REFERENCES}

Agustí S. 1991. Allometric scaling of light absorption and scattering by phytoplankton cells. Can J Fish Aquat Sci 48: 763-767.

Banse K. 1976. Rates of growth, respiration and photosynthesis of unicellular algae as related to cell size-A review. J Phycol 12: 135-140.

Banse K. 1982. Cell volumes, maximal growth rates of unicellular algae and ciliates, and the role of ciliates in the marine pelagial. Limnol Oceanogr 27: 1059-1071.

Baumberger N, Steiner M, Ryser U, Keller B, Ringli C. 2003. Synergistic interaction of the two paralogous Arabidopsis genes $L R X 1$ and $L R X 2$ in cell wall formation during root hair development. Plant J 35: 71-81.

Benkova E, Michniewicz M, Sauer M, Teichmann T, Seifertova D, Jurgens G, Friml J. 2003. Local, efflux-dependent auxin gradients as a common module for plant organ formation. Cell 115: 591-602.

Berthoumieux S, de Jong H, Baptist G, Pinel C, Ranquet C, Ropers D, Geiselmann J. 2013. Shared control of gene expression in bacteria by transcription factors and global physiology of the cell. Mole Syst Biol 9: 634.

Besson S, Dumais J. 2011. Universal rule for the symmetric division of cells. Proc Natl Acad Sci 108: 6294-6299.

Bogre L, Henriques R, Magyar Z. 2013. TOR tour to auxin. EMBO J 32: 1069-1071.

Capdevila J, Guerrero I. 1994. Targeted expression of the signaling molecule decapentaplegic induces pattern duplications and growth alterations in Drosophila wings. EMBO J 13: 4459-4468.

Cavalier-Smith T. 1982. Skeletal DNA and the evolution of genome size. Ann Rev Biophys Bioeng 11: 273-302.

Connolly JA, Oliver MJ, Beaulieu JM, Knight CA, Tomanek L, Moline MA. 2008. Correlated evolution of genome size and cell volume in diatoms (Bacillariophyceae). J Phycol 44: $124-131$.

Cosgrove DJ. 2005. Growth of the plant cell wall. Nat Rev Mol Cell Biol 6: 50-861.

Cross FR, Buchler NE, Skotheim JM. 2011. Evolution of networks and sequences in eukaryotic cell cycle control. Philos Trans R Soc Lond B Biol Sci 366: 35323544.

Csikász-Nagy A, Battogtokh D, Chen KC, Novák B, Tyson JJ. 2006. Analysis of a generic model of eukaryotic cell-cycle regulation. Biophys J 90: 4361-4379.

Day SJ, Lawrence PA. 2000. Measuring dimensions: The regulation of size and shape. Development 127: 29772987.

de Boer PAD, Crossley RE, Rothfield LI. 1989. A division inhibitor and a topological specificity factor coded for by 
K.J. Niklas

the minicell locus determine proper placement of the division septum in E. coli. Cell 56: 641-649.

Ecker JR. 1995. The ethylene signal transduction pathway in plants. Science 268: 667-675.

Eppley RW, Sloan PR. 1966. Growth rates of marine phytoplankton: Correlation with light absorption by cell chlorophyll a. Physiol Plant 19: 47-59.

Errera L. 1888. Über zellformen und siefenblasen [Concerning cell forms and soap bubbles]. Botan Central 34: 395399.

Fankhauser G. 1945a. The effects of changes in chromosome number on amphibian development. Quart Rev Biol 20: $20-78$.

Fankhauser G. 1945b. Maintenance of normal structure in heteroploid salamander larvae, through compensation of changes in cell size by adjustment of cell number and cell shape. J Exp Zool 100: 445-455.

Fantes PA, Grant WD, Pritchard RH, Sudbery PE, Wheals AE. 1975. The regulation of cell size and the control of mitosis. J Theor Biol 50: 213-244.

Foard DE, Haber AH. 1961. Atomic studies of $\gamma$-radiated wheat growing without cell division. Am J Bot 48: 438446.

Galitski T, Saldanha AJ, Styles CA, Lander ES, Fink GR. 1999. Ploidy regulation of gene expression. Science 285: $251-254$.

Gregory TR. 2001. The bigger the C-value, the larger the cell: Genome size and red blood cell size in vertebrates. Blood Cells Mol Dis 27: 830-843.

Harris M. 1971. Polyploid series of mammalian cells. Exp Cell Res 66: 329-336.

Hay A, Barkoulas M, Tsiantis M. 2006. ASYMMETRIC LEAVES1 and auxin activities converge to repress BREVIPEDICELLUS expression and promote leaf development in Arabidopsis. Development 133: 3955-3961.

Hemmingsen AM. 1960. Energy metabolism as related to body size and respiratory surfaces, and its evolution. Rep Steno Mem Hospit Nordisk Insulin Labor 9: 6-110.

Hessen DO, Jeyasingh PD, Neiman M, Weider LJ. 2010. Genome streaming and the elemental costs of growth. Trends Eco Evol 25: 75-80.

John F, Roffler S, Wicker T, Ringli C. 2011. Plant TOR signaling components. Plant Signal Behav 6: 1700-1705.

Kleiber M. 1947. Body size and metabolic rate. Physiol Rev 27: $511-541$.

Kosma SC, Thomas G. 2002. Regulation of cell size in growth, development and human disease: PI3K, PKB and S6K. BioEssays 24: 65-71.

Langdon C. 1987. On the causes of interspecific differences in the growth-irradiance relationship of phytoplankton: Part I. A comparative study of the growth-irradiance relationship of three marine phytoplankton species: Skeletonema costatum, Olisthodiscus luteus and Gonyaulax tamarensis. J Plankton Res 9: 459-482.

Langdon C. 1988. On the causes of interspecific differences in the growth-irradiance relationship of phytoplankton: Part II. A general review. J Plankton Res 10: 1291-1312.

Leevers SJ, Weinkove D, MacDougall LK, Hafen E, Waterfield MD. 1996. The Drosophila phosphoinositide 3-ki- nase Dp110 promotes cell growth. EMBO J 15: 65846594.

Levin DE. 2005. Cell wall integrity signaling in Saccharomyces cerevisiae. Microbiol Mol Biol Rev 69: 262-291.

Levin DE. 2011. Regulation of cell wall biogenesis in Saccharomyces cerevisiae: The cell wall integrity signaling pathway. Genetics 189: 1145-1175.

Lozano E, Sáez AG, Flemming AJ, Cunha A, Leroi AM. 2006. Regulation of growth by ploidy in Caenorhabditis elegans. Curr Biol 16: 493-498.

Lutkenhaus J. 2008. Min oscillation in bacteria. $A d v \operatorname{Exp}$ Med Biol 641: 1700-1705.

Mandels M, Matthern RO, El-Bisi HM. 1968. Growth of plant cell cultures: III. Growth kinetics and mass culture (Technical Report 69-22-FL). U.S. Army Natick Laboratories, Natick, MA.

Martin SG, Berthelot-Grosjean M. 2009. Polar gradients of the DYRK-family kinase Pom 1 couple cell length with the cell cycle. Nature 459: 852-856.

Mineyuki Y, Gunning BES. 1990. A role for preprophase bands of microtubules in maturation of new cell walls, and a general proposal on the function of preprophase band sites in cell division in higher plants. J Cell Sci 97: 527-537.

Mitchison JM. 2003. Growth during the cell cycle. Internatl Rev Cytol 226: 165-258.

Mizukami Y. 2001. A matter of size: Developmental control of organ size in plants. Curr Opin Cell Biol 4: 533-539.

Mizukami Y, Fischer RL. 2000. Plant organ size control: AINTEGUMENTA regulates growth and cell numbers during organogenesis. Proc Natl Acad Sci 97: 942-947.

Moseley JB, Mayeux A, Paoletti A, Nurse P. 2009. A spatial gradient coordinates cell size and mitotic entry in fission yeast. Nature 459: 857-860.

Mullin MM, Sloan PR, Eppley RW. 1966. Relationship between carbon content, cell volume, and area in phytoplankton. Limnol Ocean 11: 307-311.

Murata T, Wada M. 1991. Effects of centrifugation on preprophase-band formation in Adiantum protonemata. Planta 183: 391-398.

Niklas KJ. 1994a. Size-dependent variations in plant growth rates and the "3/4-power rule." Am J Bot 81: 134-144.

Niklas KJ. 1994b. Plant allometry: The scaling of form and process. University of Chicago Press, Chicago.

Niklas KJ, Enquist BJ. 2001. Invariant scaling relationships for interspecific plant biomass production rates and body size. Proc Natl Acad Sci 98: 2922-2927.

Niklas KJ, Newman SA. 2013. The origins of multicellular organisms. Evol Dev 15: 41-52.

Olmo E. 1983. Nucleotype and cell size in vertebrates: A review. Bas Appl Histochem 27: 227-256.

Potter CJ, Xu T. 2001. Mechanisms of size control. Curr Opin Gen Devel 11: 279-286.

Roeder AHK. 2012. When and where plant cells divide: A perspective from computational modeling. Curr Opin Plant Biol 15: 638-644.

Rothfield L, Taghbalout A, Shih YL. 2005. Spatial control of bacterial division-site placement. Nat Rev Microbiol 3: 959-968. 
Phyletic Perspective on Cell Growth

Sachs J. 1892. Beiträge zur Zellentheorie. Energiden und Zellen [Contribution to cell theory: Syncytia and cells]. Flora 75: 57-67.

Satina S, Blakeslee AF, Avery AG. 1940. Demonstration of the three germ layers in the shoot apex of Datura by means of induced polyploidy in periclinal chimeras. Am J Bot 27: 895-905.

Shuter BJ, Thomas JE, Taylor WD, Zimmerman AM. 1983. Phenotypic correlates of genomic DNA content in unicellular eukaryotes and other cells. Am Nat 122: 26-44.

Simova I, Herben T. 2012. Geometrical constraints in the scaling relationships between genome size, cell size and cell cycle length in herbaceous plants. Proc Roy Soc Biol Sci 279: 867-875.

Strome S, Wood WB. 1983. Generation of asymmetry and segregation of germ-line granules in early C. elegans embryos. Cell 35: 15-25.

Taguchi S. 1976. Relationship between photosynthesis and cell-size of marine diatoms. J Phycol 12: 185-189.

Tang EPY. 1995. The allometry of algal growth rates. J Plank Res 17: 1325-1335.

Tsukaya H. 2003. Organ shape and size: A lesson from studies of leaf morphogenesis. Curr Opin Plant Biol 6: 57-62.
Vervoort M, Crozatier M, Valle D, Vincent A. 1999. The COE transcription factor Collier is a mediator of short-range Hedgehog-induced patterning of the Drosophila wing. Curr Biol 9: 632-639.

von Dassow P, Chepurnov VA, Armbrust EV. 2006. Relationships between growth rate, cell size, and induction of spermatogenesis in the centric diatom Thalassiosira weissflogii (Bacillariophyta). J Phycol 42: 887-899.

von Dassow P, Petersen TW, Timothy W, Chepurnov VA, Armbrust EV. 2008. Inter- and intraspecific relationships between nuclear DNA content and cell size in selected members of the centric diatom genus Thalassiosira (Bacillariophyta). J Phycol 44: 335-349.

Wilcox M, Mitchison CJ, Smith RJ. 1973. Pattern formation in the blue-green alga Anabaena: I. Basic mechanisms. J Cell Sci 12: 707-723.

Williams RB. 1964. Division rates of Salt Marsh diatoms in relation to salinity and cell size. Ecology 45: 877-880.

Wullschleger S, Loewith R, Hall MN. 2006. TOR signaling in growth and metabolism. Cell 124: 471-484.

Xu T, Wang W, Zhang S, Stewart RA, Yu W. 1995. Identifying tumor suppressors in genetic mosaics: The Drosophila lats gene encodes a putative protein kinase. Development 121: 1053-1063. 


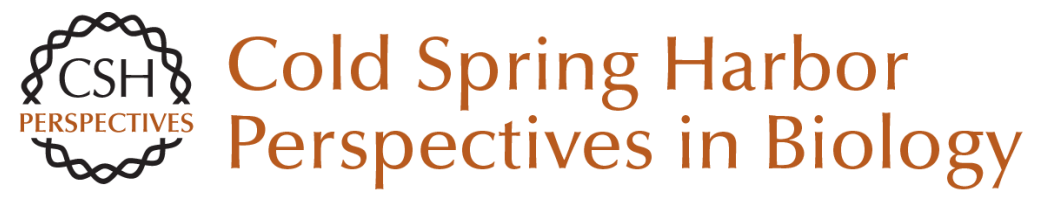

\section{A Phyletic Perspective on Cell Growth}

Karl J. Niklas

Cold Spring Harb Perspect Biol 2015; doi: 10.1101/cshperspect.a019158

Subject Collection Size Control in Biology: From Organelles to Organisms

\section{Cell-Size Control}

Amanda A. Amodeo and Jan M. Skotheim

Indeterminate Growth: Could It Represent the

Ancestral Condition?

Iswar K. Hariharan, David B. Wake and Marvalee

H. Wake

The Systemic Control of Growth

Laura Boulan, Marco Milán and Pierre Léopold

Genome Biology and the Evolution of Cell-Size

Diversity

Rachel Lockridge Mueller

Size Scaling of Microtubule Assemblies in Early

Xenopus Embryos

Timothy J. Mitchison, Keisuke Ishihara, Phuong Nguyen, et al.

The Influence of Genome and Cell Size on Brain

Morphology in Amphibians

Gerhard Roth and Wolfgang Walkowiak

The Opposing Actions of Target of Rapamycin and AMP-Activated Protein Kinase in Cell Growth Control

Sravanth K. Hindupur, Asier González and Michael N. Hall

Small but Mighty: Cell Size and Bacteria

Petra Anne Levin and Esther R. Angert
Mechanical Forces and Growth in Animal Tissues Loïc LeGoff and Thomas Lecuit

Biological Scaling Problems and Solutions in Amphibians

Daniel L. Levy and Rebecca Heald

Intracellular Scaling Mechanisms Simone Reber and Nathan W. Goehring

Growing an Embryo from a Single Cell: A Hurdle

in Animal Life

Patrick H. O'Farrell

Organ-Size Regulation in Mammals

Alfredo I. Penzo-Méndez and Ben Z. Stanger

Size Control in Plants--Lessons from Leaves and Flowers

Hjördis Czesnick and Michael Lenhard

Nuclear DNA Content Varies with Cell Size across

Human Cell Types

James F. Gillooly, Andrew Hein and Rachel

Damiani

Subcellular Size

Wallace F. Marshall

For additional articles in this collection, see http://cshperspectives.cshlp.org/cgi/collection/

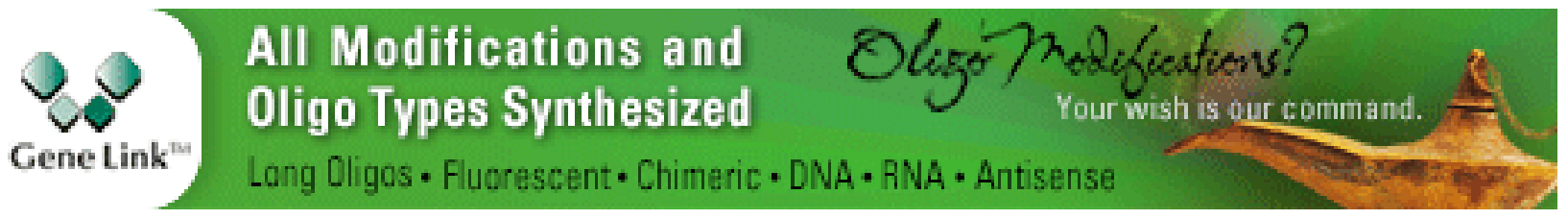

Copyright @ 2015 Cold Spring Harbor Laboratory Press; all rights reserved 